\title{
Antiproliferative and antibacterial activity of some glutarimide derivatives
}

Jelena B. Popović-Djordjević, Anita S. Klaus, Željko S. Žižak, Ivana Z. Matić \& Branko J. Drakulić

To cite this article: Jelena B. Popović-Djordjević, Anita S. Klaus, Željko S. Žižak, Ivana Z.

Matić \& Branko J. Drakulić (2016) Antiproliferative and antibacterial activity of some glutarimide derivatives, Journal of Enzyme Inhibition and Medicinal Chemistry, 31:6, 915-923, DOI:

10.3109/14756366.2015.1070844

To link to this article: https://doi.org/10.3109/14756366.2015.1070844

View supplementary material

Published online: 06 Aug 2015.

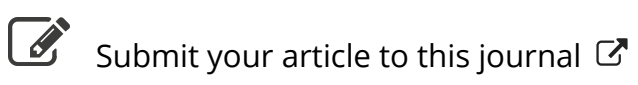

Џ Article views: 525

View Crossmark data $\nearrow$

Citing articles: 3 View citing articles $๘$ 


\title{
Antiproliferative and antibacterial activity of some glutarimide derivatives
}

\author{
Jelena B. Popović-Djordjević ${ }^{1}$, Anita S. Klaus ${ }^{2}$, Željko S. Žižak ${ }^{3}$, Ivana Z. Matić ${ }^{3}$, and Branko J. Drakulić ${ }^{4}$ \\ ${ }^{1}$ Department of Chemistry and Biochemistry and ${ }^{2}$ Department for Industrial Microbiology, Faculty of Agriculture, University of Belgrade, Belgrade, \\ Serbia, ${ }^{3}$ Institute of Oncology and Radiology of Serbia, Belgrade, Serbia, and ${ }^{4}$ Department of Chemistry, Institute of Chemistry, Technology and \\ Metallurgy, University of Belgrade, Belgrade, Serbia
}

\section{Abstract}

Antiproliferative and antibacterial activities of nine glutarimide derivatives (1-9) were reported. Cytotoxicity of compounds was tested toward three human cancer cell lines, HeLa, K562 and MDA-MB-453 by MTT assay. Compound 7 (2-benzyl-2-azaspiro[5.11]heptadecane-1,3,7-trione), containing 12-membered ketone ring, was found to be the most potent toward all tested cell lines $\left(\mathrm{IC}_{50}=9-27 \mu \mathrm{M}\right)$. Preliminary screening of antibacterial activity by a disk diffusion method showed that Gram-positive bacteria were more susceptible to the tested compounds than Gram-negative bacteria. Minimum inhibitory concentration (MIC) determined by a broth microdilution method confirmed that compounds 1, 2, 4, 6-8 and $\mathbf{9}$ inhibited the growth of all tested Gram-positive and some of the Gram-negative bacteria. The best antibacterial potential was achieved with compound 9 (ethyl 4-(1-benzyl-2,6-dioxopiperidin-3-yl)butanoate) against Bacillus cereus (MIC $0.625 \mathrm{mg} / \mathrm{mL} ; 1.97 \times 10^{-3} \mathrm{~mol} / \mathrm{L}$ ). Distinction between more and less active/ inactive compounds was assessed from the pharmacophoric patterns obtained by molecular interaction fields.
\end{abstract}

\section{Introduction}

Both naturally occurring and synthetic cyclic imides, especially five- and six-membered systems, are an important group of bioactive molecules. They exhibit widespread pharmacological effects, including antitumor ${ }^{1-4}$, anti-inflammatory ${ }^{5}$, immunomodulatory, antiangiogenic and anxiolytic ${ }^{6-8}$.

Isolation and examination of pharmacologically active natural glutarimides started in 1960s. Initially, cycloheximide ${ }^{9}$ and streptimidone $^{10-12}$ were examined as antibiotics, but later it was found that they acted as very potent cytotoxic agents ${ }^{13,14}$. The structurally related streptimidone derivative, 9-methylstreptimidone, exerts a significant inhibitory activity toward nuclear

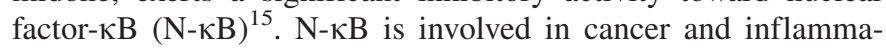
tions. Alkaloids (+)-sesbanimide A and (-)-sesbanimide B were isolated from the seeds of the leguminous plant Sesbania drummondii $^{1,16}$. Ethanol extracts of $S$. drummondii seeds showed significant inhibitory activity against P388 murine leukemia model in mice (in vivo) ${ }^{17-19}$. Structurally related natural product lactimidomycin (LTM), 12-membered unsaturated macrolide antibiotic that comprise biosynthetically rare glutarimide side chain, produced by Streptomyces amphibiosporus R310-104 (ATCC 53964), display strong cytotoxicity against a number of human tumor cell lines in vitro, in vivo antitumor activity in mice model and potent antifungal activity ${ }^{2}$.

Address for correspondence: Jelena B. Popović-Djordjević, Department of Chemistry and Biochemistry, Faculty of Agriculture, University of Belgrade, Nemanjina 6, 11080 Belgrade, Serbia. E-mail: jelenadj@ agrif.bg.ac.rs

\section{Keywords \\ Antitumor agents, heterocycles, structure-activity analysis}

\section{History}

Received 7 May 2015

Revised 25 June 2015

Accepted 26 June 2015

Published online 6 August 2015
In 1990s it was discovered that thalidomide, a well-known synthetic glutarimide derivative, has anti-inflammatory and antiangiogenic properties. It was approved as a drug for the treatment of certain cancers (newly diagnosed multiple myeloma) and for complication arised from leprosy. Later on, analogs of thalidomide with increased potency, 3-amino-thalidomid (pomalidomid, Pomalyst) and $\alpha$-(3-aminophthalimido) glutarimide (lenalidomid, Revlimid) have been developed ${ }^{20,21}$. Lenalidomid has been used for the treatment of multiple myeloma, while pomalidomid has been recently approved by FDA for the treatment of relapsed and refractory multiple myeloma ${ }^{22}$.

Some estrone derivatives with the D-ring replaced with the glutarimide moiety showed potent inhibition of steroid sulfatase, an enzyme involved in the pathway of the development of hormone-dependent breast tumors ${ }^{23}$; while aminoglutethimide, the non-steroidal aromatase inhibitor, is in use for the treatment of hormone-sensitive metastatic breast cancer $^{24,25}$. In the past decade, antitumor activity in vitro of mitonafide ${ }^{26}$, amonafide ${ }^{27}$ and naphthalimide ${ }^{28,29}$ derivatives was intensively examined.

Upon detail analysis of bioactive compounds that comprise glutarimide moiety in their structure, as is briefly outlined in previous paragraphs, we concluded that data on antiproliferative and on antibacterial activity of compounds 1-9 (Figure 1) cannot be found in literature. Those compounds have been prepared in our group to demonstrate novel synthetic approach of glutarimide ring closure reaction $^{30}$. All compounds have a common $N$-substituted glutarimide moiety in their structure, and bear structurally diverse substituents in positions 3 and/or 4 of glutarimide ring. We have tested antiproliferative and antibacterial activity of compounds 1-9 in vitro, and hereby report on the results obtained. 
Figure 1. Structures of glutarimide derivatives 1-9.<smiles>O=C1CCCC(=O)N1Cc1ccccc1</smiles>

1<smiles>CCC(C)(C)OC(=O)[C@H]1C(=O)N(Cc2ccccc2)C(=O)C[C@H]1C</smiles>

5

mixture of cis/trans isomers (1:5)<smiles>CCCCCCCCCCCCCCCCCCC12CCC(=O)C(CCC1=O)N(Cc1ccccc1)C2=O</smiles>

7<smiles>O=C1CC2(CCCC2)CC(=O)N1Cc1ccccc1</smiles><smiles>N#C[C@H]1CCC(=O)N(Cc2ccccc2)C1=O</smiles><smiles>CCCN1C(=O)CC[C@H](C(=O)OC(C)(C)CC)C1=O</smiles><smiles>CC(c1ccccc1)N1C(=O)CC[C@H](S(=O)(=O)c2ccccc2)C1=O</smiles>

mixture of two diasteroisomers (1:1)<smiles>O=C1CCC2(CCCCCCC2=O)C(=O)N1Cc1ccccc1</smiles>

8<smiles>CCOC(=O)CCC[C@H]1CCC(=O)N(Cc2ccccc2)C1=O</smiles>

9

\section{Methods}

\section{Antiproliferative activity}

Stock solutions of investigated compounds were prepared in a nutrient medium (RPMI-1640) supplemented with $3 \mathrm{mM}$ L-glutamine, $100 \mu \mathrm{g} / \mathrm{mL}$ streptomycin, $100 \mathrm{IU} / \mathrm{mL}$ penicillin, $10 \%$ heat inactivated fetal bovine serum (FBS) and $25 \mathrm{mM}$ Hepes, adjusted to $\mathrm{pH} 7.2$ by bicarbonate solution. RPMI-1640, FBS, Hepes and L-glutamine were products of Sigma Chemical Co., St. Louis, MO. Human cervix adenocarcinoma HeLa, breast carcinoma MDA-MB-453 and normal lung fibroblast MRC-5 cells were cultured as monolayers in the nutrient medium, while human myelogenous leukemia K562 cells were maintained as suspension culture. The cells were grown at $37{ }^{\circ} \mathrm{C}$ in $5 \% \mathrm{CO}_{2}$ and humidified air atmosphere.

HeLa (2000 cells per well), MDA-MB-453 (3000 c/w) and MRC-5 $(5000 \mathrm{c} / \mathrm{w})$ cells were seeded into 96-well microtiter plates and $20 \mathrm{~h}$ later, after the cell adherence, five different concentrations of investigated compounds were added to the wells. Final concentrations were in the range from 200 to $12.5 \mu \mathrm{M}$. Only nutrient medium was added to the cells in the control wells. Investigated compound was added to a suspension of leukemia K562 cells (5000 cells/ well) $2 \mathrm{~h}$ after cell seeding, in the same final concentrations applied to HeLa, MDA-MB-453 and MRC-5 cells. All experiments were done in triplicate. Nutrient medium with corresponding concentrations of compound, but void of cells, was used as blank.

Cell survival was determined by MTT test according to the method of Mosmann and modified by Ohno and Abe, $72 \mathrm{~h}$ after addition of the compounds ${ }^{31,32}$. Briefly, $20 \mu \mathrm{L}$ of MTT solution $(5 \mathrm{mg} / \mathrm{mL}$ in phosphate buffered saline) was added to each well. Samples were incubated for further $4 \mathrm{~h}$ at $37^{\circ} \mathrm{C}$ in humidified atmosphere with $5 \% \mathrm{CO}_{2}$. Then, $100 \mu \mathrm{L}$ of $10 \%$ SDS was added to the wells. Absorbance was measured at $570 \mathrm{~nm}$ the next day. To achieve cell survival (S\%), absorbance at $570 \mathrm{~nm}$ of a sample with cells grown in the presence of various concentrations of compounds tested was divided with absorbance of control sample (the absorbance of cells grown in nutrient medium only). Absorbance of blank was always subtracted from absorbance of a corresponding sample with cells. All experimentally obtained $\mathrm{IC}_{50}$ data were means of three measurements done in triplicate.

\section{The antibacterial activity testing}

The antibacterial activity of compounds 1, 2, 4-8 and 9 was determined against four Gram-positive (Staphylococcus aureus, Enterococcus faecalis, Bacillus cereus, Listeria monocytogenes) and seven Gram-negative bacterial species [Pseudomonas aeruginosa, Escherichia coli, Salmonella enteritidis, Proteus hauseri, Shigella sonnei, Yersinia enterocolitica, E. coli $(\mathrm{O} 157: \mathrm{H} 7)]$. Selected bacterial strains originated from ATCC (American Type Culture Collection, Rockville, MD). These microorganisms were chosen for the bioassay as the well-known food spoilage and pathogenic bacteria. Each species was maintained on Mueller-Hinton agar (MHA), which was also used to confirm the absence of contamination and the validity of the inocula. Before testing, each species was recovered by subculturing in Mueller-Hinton broth (MHB), aerobically, for $24 \mathrm{~h}$, at $37^{\circ} \mathrm{C}$. Working concentrations of approximately $10^{5}-10^{6} \mathrm{cfu} /$ $\mathrm{mL}$, used for antibacterial activity assays, were prepared by proper dilution of culture in microbiological medium. Compounds were dissolved in DMSO (2\%) to prepare stock solutions at a concentration of $40 \mathrm{mg} / \mathrm{mL}$, sterilized by filtration through a $0.22-\mu \mathrm{m}$ membrane filter (Sartorius AG - Göttingen, Germany) according to Tepe et al. and further diluted in MHB to a working solutions. DMSO was chosen as a non-toxic solvent ${ }^{33}$. 
Disk diffusion assay was performed using a slightly modified $\mathrm{CLSI}^{34}$. Each bacterial culture (approximately $10^{5}-10^{6} \mathrm{cfu} / \mathrm{mL}$ ) was added $(0.1 \mathrm{~mL})$ to Petri dishes $(90 \mathrm{~mm})$ containing MHA $(20 \mathrm{~mL})$. Three sterile blank paper disks $(6 \mathrm{~mm}$ in diameter, Susceptibility Test Discs, SD 067-5CT, HiMedia, Mumbai, India) were placed on the surface of each agar plate and inoculated with $10 \mu \mathrm{L}$ of the compound $(20 \mathrm{mg} / \mathrm{mL})$. After $2 \mathrm{~h}$ at $25^{\circ} \mathrm{C}$, the plates were incubated aerobically, for $24 \mathrm{~h}$ at $37^{\circ} \mathrm{C}$. After incubation period, inhibition zone $(\mathrm{mm})$ was measured including the initial diameter of the disk. Tests were performed in triplicate and the results were analyzed for statistical significance. The plates with MHA were sterility controls. Negative controls were disks impregnated with DMSO. As positive controls disks (SigmaAldrich GmbH, Steinheim, Germany) with gentamicin $(30 \mu \mathrm{g})$ and tetracycline $(30 \mu \mathrm{g})$ were used.

Broth microdilution method was employed to determine minimum inhibitory concentrations (MICs) ${ }^{34,35}$. Concentrations of compound ranged from 10.0 to $0.048 \mathrm{mg} / \mathrm{mL}$. Test bacterial culture $(50 \mu \mathrm{L})$ in a MHB was added to the wells of a sterile 96well microtiter plate (Sarstedt, Numbrecht, Germany) already containing $50 \mu \mathrm{L}$ of twofold serially diluted compound in MHB. The final volume in each well was $100 \mu \mathrm{L}$. The microplates were prepared in triplicate and incubated aerobically, for $24 \mathrm{~h}$ at $37^{\circ} \mathrm{C}$. Wells with MHB was used as a sterility control, while negative controls were wells with tested compound in $50 \mu \mathrm{L}$ of MHB, but void of bacteria. Positive controls were wells with a bacterial suspension in $50 \mu \mathrm{L}$ of MHB and wells with a bacterial suspension in a MHB with DMSO, in amounts corresponding to the highest quantity present in the broth microdilution assay (to prove that DMSO had no inhibition effect on the bacterial growth). A microplate shaker (Lab Companion, VM-96B, Seoul, South Korea) was used for mixing the content of each well at $900 \mathrm{rpm}$ for $1 \mathrm{~min}$ prior to incubation in the cultivation conditions described above. To indicate cellular respiration 2,3,5-triphenyltetrazolium chloride (TTC) (Aldrich Chemical Company Inc., Sigma-Aldrich, St. Louis, MO) was added to the culture medium. The final concentration of TTC after inoculation was $0.05 \%$. Viable microorganisms enzymatically reduced white TTC to a pink TPF (1,3,5-triphenylformazan). The MIC was defined as the lowest sample concentration that prevented this change and exhibited complete inhibition of bacterial growth.

All measurements were done in triplicate and data were expressed as mean \pm standard deviation. The experimental data were subjected to an one-way analysis of variance (ANOVA) and Fisher's LSD was calculated to detect significant difference $(p \leq 0.05)$ between the mean values.

\section{Molecular modeling}

Initial 3D structures of compounds 1-9 were generated from SMILES notation in CORINA assuming $R$ stereochemistry for all stereogenic centers, except for $\mathrm{C} 2$ of the phenethyl moiety of compound $6^{36,37}$. The $S$ stereochemistry was ascribed to this stereogenic center, on the ground of experimental data ${ }^{30}$. Initial structures were imported in VegaZZ ${ }^{38}$. Up to 20 conformations, representing local energy minima, were obtained by conformational search on the molecular mechanics level (MMFF94s force field), using Boltzmann jump algorithm in $\mathrm{AMMP}^{39,40}$. Each conformation of each compound was minimized by the semiempirical molecular orbital PM6 method, using implicit solvation in water (COSMO) to root mean square gradient of 0.01 ; by MOPAC2012 $2^{41,42}$. Conformation of each compound that had the lowest heat of formation (implying the most stable one) was chosen for further modeling. 3D-dependent whole-molecular properties of compounds, surface area, polar surface area, apolar surface area, volume and virtual $\log P$, were calculated in VegaZZ, using $1.4 \AA$ probe $^{43}$. Molecular interaction fields (MIF) around molecules were calculated by a GRID method, as applied in Pentacle program, using grid resolution of $0.4 \AA^{44}$. Hydrogenbond donor (N1), hydrogen-bond acceptor (O), hydrophobic (DRY) and shape (TIP) probes were used. AMANDA algorithm were used for the extraction of hot spots (nodes) from the obtained MIFs (discretization); the distances and relative position of the nodes were described by maximum auto- and cross-correlation (MACC2) (encoding). For more exhaustive description of applied methodology see original reference ${ }^{45}$. Auto- and crosscorrelograms, obtained by the Pentacle program are depicted in matrix-like representation, named as heatmap. Values of variables are color-coded from red (low value) to blue (high value). For color code in heatmap, depicted in Figure 3, see on-line version of the article. Correlograms encode molecular descriptors grounded on two-point pharmacophoric pattern, which represent the local minima of two probes (nodes) around molecule, including distance between those nodes. All blocks of correlograms (DRY-DRY, O-O, N1-N1, TIP-TIP, DRY-O, DRYN1, DRY-TIP, O-N1, O-TIP, N1-TIP) were considered during analysis.

\section{Chemistry}

Chemicals and solvents were purchased from Merck (Darmstadt, Germany), Sigma-Aldrich and Fluka (Basel, Switzerland). All reagents were of analytically pure. All solvents were dried by standard methods and distilled before use. The sodium hydride was used as a $60 \%$ dispersion in mineral oil. 18-Crown-6 ether was prepared according to a literature procedure ${ }^{46}$. Reactions were monitored on silica gel precoated TLC plates, $\mathrm{HF}_{254}$ (Merck). The dry-flash chromatography on silica gel $(12-16 \mu$, ICN Pharmaceuticals, Costa Mesa, CA) was used to purify the reaction products. Anhydrous reactions were carried out in ovendried glassware in extra pure argon atmosphere. ${ }^{1} \mathrm{H}$ and ${ }^{13} \mathrm{C}$ NMR spectra were recorded on a Bruker Avance 500 (Bruker BioSpin GmbH, Karlsruhe, Germany), or Varian (Palo Alto, CA) Gemini 2000 instruments on $500 / 125$ or $200 / 50 \mathrm{MHz}$, in $\mathrm{CDCl}_{3}$ with TMS as an internal reference. ESI-MS spectra were recorded on Agilent Technologies (Santa Clara, CA) 6210-1210 TOF-LC-ESI-HR/MS instrument in positive mode. Integrity and purity of all compounds used for biological tests are routinely checked by NMR and ESI/HR-MS.

\section{General procedure for the synthesis of compounds $\mathbf{1}$ and $\mathbf{2}$}

1-Benzyl-piperidine-2,6-dione $\left(1, \mathrm{C}_{12} \mathrm{H}_{13} \mathrm{NO}_{2}\right)$. Toluene $(20 \mathrm{~mL})$ and sodium hydride $(2.9 \mathrm{~g}, 72 \mathrm{mmol})$ were placed in a two-necked flask equipped with reflux condenser and dropping funnel. A solution of 4-benzylcarbamoyl-butyric acid methyl ester $\mathbf{1 c}$ $(5.0 \mathrm{~g}, 18 \mathrm{mmol})$ in toluene $(\sim 30 \mathrm{ml})$ was added and mixture was refluxed for $3 \mathrm{~h}$. After cooling at room temperature the mixture was filtered and the filtrate was evaporated under reduced pressure to give $65 \%$ of compound $1\left(\mathrm{C}_{12} \mathrm{H}_{13} \mathrm{NO}_{2}\right) .{ }^{1} \mathrm{H} \mathrm{NMR}$ $\left(200 \mathrm{MHz}, \mathrm{CDCl}_{3}\right): \delta=7.36-7.24(\mathrm{~m}, 5 \mathrm{H}, \mathrm{Ar}-\mathrm{H}), 4.94(\mathrm{~s}, 2 \mathrm{H}$; $\mathrm{CH}_{2}$ ), 2.65 (t, $\left.J=6.5 \mathrm{~Hz}, 4 \mathrm{H}, \mathrm{CH}_{2}\right), 1.98-1.88\left(\mathrm{~m}, 2 \mathrm{H}, \mathrm{CH}_{2}\right) \mathrm{ppm}$; ${ }^{13} \mathrm{C} \mathrm{NMR}\left(50 \mathrm{MHz}, \mathrm{CDCl}_{3}\right): \delta=172.5\left(\mathrm{CCO}_{\text {imide }}\right),[137.3,128.8$, 128.4, $\left.127.4\left(\mathrm{C}_{\mathrm{Ar}}\right)\right], 42.6\left(\mathrm{CCH}_{2}\right), 32.9\left(\mathrm{CCH}_{2}\right), 17.0(\mathrm{CCH}) \mathrm{ppm}$; HR-MS (ESI, $m / z)$ : calcd. for $\mathrm{C}_{12} \mathrm{H}_{13} \mathrm{NO}_{2}\left[M+\mathrm{NH}_{4}\right]^{+}$: 221.1284; found: 221.1278 .

8-Benzyl-8-aza-spiro[4.5]decane-7,9-dione (2, $\left.\mathrm{C}_{16} \mathrm{H}_{19} \mathrm{NO}_{2}\right)$. Prepared from methyl ester 2e. Yield $75 \%$; ${ }^{1} \mathrm{H}$ NMR $(200 \mathrm{MHz}$, $\left.\mathrm{CDCl}_{3}\right): \delta=7.37-7.24(\mathrm{~m}, 5 \mathrm{H}, \mathrm{Ar}-\mathrm{H}), 4.94\left(\mathrm{~s}, 2 \mathrm{H}, \mathrm{CH}_{2}\right), 2.60$ (s, 4H, $\left.\mathrm{CH}_{2}\right), 1.72-1.65\left(\mathrm{~m}, 4 \mathrm{H}, \mathrm{CH}_{2}\right), 1.50-1.44\left(\mathrm{~m}, 4 \mathrm{H}, \mathrm{CH}_{2}\right)$ ppm; ${ }^{13} \mathrm{C}$ NMR $\left(50 \mathrm{MHz}, \mathrm{CDCl}_{3}\right): \delta=172.1\left(\mathrm{CCO}_{\text {imide }}\right),[137.2$, 
128.5, 128.3, $\left.127.3\left(\mathrm{C}_{\mathrm{Ar}}\right)\right], 44.7\left(\mathrm{CCH}_{2}\right), 42.6\left(\mathrm{CCH}_{2}\right), 39.4(\mathrm{C})$, $37.4\left(\mathrm{CCH}_{2}\right), 24.1\left(\mathrm{CCH}_{2}\right)$ ppm; HR-MS (ESI, $\left.\mathrm{m} / \mathrm{z}\right)$ : calcd. for $\mathrm{C}_{16} \mathrm{H}_{19} \mathrm{NO}_{2}[M+\mathrm{H}]^{+}$: 258.1489; found: 258.1480 .

General procedure described in our previous paper was used for preparation of compounds $\mathbf{3}-\mathbf{9}$

2,6-Dioxo-1-phenethylpiperidine-3-carbonitrile $\left(3, \mathrm{C}_{14} \mathrm{H}_{14} \mathrm{~N}_{2} \mathrm{O}_{2}\right)$. Yield 65\%; ${ }^{1} \mathrm{H}$ NMR $\left(200 \mathrm{MHz}, \mathrm{CDCl}_{3}\right): \delta=7.34-7.19(\mathrm{~m}, 5 \mathrm{H}$, Ar-H), 4.05 (splitted t, $J=7.0 \mathrm{~Hz} ; J=2.1 \mathrm{~Hz}, 2 \mathrm{H}, \mathrm{CH}_{2}$ ), 3.71 (dd, $J=9.3 \mathrm{~Hz}, J=5.3 \mathrm{~Hz}, 1 \mathrm{H}, \mathrm{CH}), 2.95-2.81\left(\mathrm{~m}, 3 \mathrm{H}, \mathrm{CH}_{2}\right), 2.72-$ $2.56\left(\mathrm{~m}, 1 \mathrm{H}, \mathrm{CH}_{2}\right), 2.36-2.17\left(\mathrm{~m}, 2 \mathrm{H}, \mathrm{CH}_{2}\right) \mathrm{ppm} ;{ }^{13} \mathrm{C} \mathrm{NMR}$ $\left(50 \mathrm{MHz}, \mathrm{CDCl}_{3}\right): \delta=169.6\left(\mathrm{CCO}_{\text {imide }}\right),[137.7,129.0,128.5$, $\left.126.7\left(\mathrm{C}_{\mathrm{Ar}}\right)\right], 115.1(\mathrm{CCN}), 41.6\left(\mathrm{CCH}_{2}\right), 35.7\left(\mathrm{CCH}_{2}\right), 33.6$ $(\mathrm{CCH}), 30.7\left(\mathrm{CCH}_{2}\right), 21.5\left(\mathrm{CCH}_{2}\right)$ ppm; HR-MS (ESI, $\left.\mathrm{m} / z\right)$ : calcd. for $\mathrm{C}_{14} \mathrm{H}_{14} \mathrm{~N}_{2} \mathrm{O}_{2}\left[M+\mathrm{NH}_{4}\right]^{+}: 260.1393$, found: $260.1389 .^{30}$

Tert-pentyl-2,6-dioxo-1-phenethylpiperidine-3-carboxylate (4, $\left.\mathrm{C}_{19} \mathrm{H}_{25} \mathrm{NO}_{4}\right)$. Yield $70 \%$; ${ }^{1} \mathrm{H}$ NMR $\left(200 \mathrm{MHz}, \mathrm{CDCl}_{3}\right) \delta=7.30$ $7.21(\mathrm{~m}, 5 \mathrm{H}, \mathrm{Ar}-\mathrm{H}), 4.04-3.96\left(\mathrm{~m}, 2 \mathrm{H}, \mathrm{CH}_{2}\right), 3.57-3.51(\mathrm{~m}, 1 \mathrm{H}$, $\mathrm{CH}), 2.86-2.78\left(\mathrm{~m}, 2 \mathrm{H}, \mathrm{CH}_{2}\right), 2.67(\mathrm{dt}, J=11.6 \mathrm{~Hz}, J=5.8 \mathrm{~Hz}$, $2 \mathrm{H}, \mathrm{CH}_{2}$ ), 2.25-2.07 (m, 2H, $\left.\mathrm{CH}_{2}\right), 1.80$ (q, $J=7.5 \mathrm{~Hz}, 2 \mathrm{H}, \mathrm{CH}_{2}$ ), $1.46\left(\mathrm{~s}, 6 \mathrm{H}, \mathrm{CH}_{3}\right), 0.90\left(\mathrm{t}, J=7.5 \mathrm{~Hz}, 3 \mathrm{H}, \mathrm{CH}_{3}\right) \mathrm{ppm} ;{ }^{13} \mathrm{C} \mathrm{NMR}$ $\left(50 \mathrm{MHz}, \mathrm{CDCl}_{3}\right): \delta=171.3\left(\mathrm{CCO}_{\text {imide }}\right), 168.8\left(\mathrm{CCO}_{\text {imide }}\right), 167.7$ $\left(\mathrm{CCO}_{\text {ester }}\right),\left[138.4,128.9,126.4\left(\mathrm{C}_{\mathrm{Ar}}\right)\right], 85.6(\mathrm{C}), 50.0(\mathrm{CCH})$, $41.1\left(\mathrm{CCH}_{2}\right), 33.8\left(\mathrm{CCH}_{2}\right), 33.3\left(\mathrm{CCH}_{2}\right), 30.8\left(\mathrm{CCH}_{2}\right), 30.2$ $\left(\mathrm{CCH}_{2}\right), 25.3\left(\mathrm{CCH}_{3}\right), 20.7\left(\mathrm{CCH}_{2}\right), 8.1\left(\mathrm{CCH}_{3}\right)$ ppm; HR-MS (ESI, $m / z$ ): calcd. for $\mathrm{C}_{19} \mathrm{H}_{25} \mathrm{NO}_{4}[M+\mathrm{H}]^{+}$: 332.1856 , found: 332.1841 .

Tert-pentyl-1-benzyl-4-methyl-2,6-dioxopiperidine-3-carboxylate $\left(5, \mathrm{C}_{19} \mathrm{H}_{25} \mathrm{NO}_{4}\right)$. Yield 46.6\%; $\left.{ }^{1} \mathrm{H} \mathrm{NMR} \mathrm{(200} \mathrm{MHz}, \mathrm{CDCl}_{3}\right)$ : $\delta=7.38-7.24(\mathrm{~m}, 5 \mathrm{H}, \mathrm{Ar}-\mathrm{H}), 5.04-4.88\left(\mathrm{~m}, 2 \mathrm{H}, \mathrm{CH}_{2}\right), 3.59$ $(\mathrm{d}, J=4.9 \mathrm{~Hz}, 0.13 \mathrm{H}, \mathrm{CH}), 3.21(\mathrm{~d}, J=9.0 \mathrm{~Hz}, 0.59 \mathrm{H}, \mathrm{CH}), 2.83$ (dd, $J=16.5, J=3.9 \mathrm{~Hz}, 1 \mathrm{H}, \mathrm{CH}), 2.74-2.68\left(\mathrm{~m}, 0.41 \mathrm{H}, \mathrm{CH}_{2}\right)$, 2.60-2.46 (m, 1H, $\left.\mathrm{CH}_{2}\right), 2.43-2.29\left(\mathrm{~m}, 1 \mathrm{H}, \mathrm{CH}_{2}\right), 1.76$ (dt, $\left.J=9.4 \mathrm{~Hz}, J=4.8 \mathrm{~Hz}, 2 \mathrm{H}, \mathrm{CH}_{2}\right), 1.44\left(\mathrm{~s}, 5 \mathrm{H}, \mathrm{CH}_{3}\right), 1.36$ $\left(\mathrm{d}, J=3.5 \mathrm{~Hz}, 1 \mathrm{H}, \mathrm{CH}_{3}\right), 1.14-1.07\left(\mathrm{~m}, 3 \mathrm{H}, \mathrm{CH}_{3}\right), 0.91-0.75(\mathrm{~m}$, $\left.3 \mathrm{H}, \mathrm{CH}_{3}\right) \quad \mathrm{ppm} ;{ }^{13} \mathrm{C} \quad \mathrm{NMR} \quad\left(50 \mathrm{MHz}, \mathrm{CDCl}_{3}\right): \quad \delta=170.9$ $\left(\mathrm{CCO}_{\text {imide }}\right), 169.1\left(\mathrm{CCO}_{\text {imide }}\right), 167.4\left(\mathrm{CCO}_{\text {ester }}\right),[136.9,128.7$, 128.4, $\left.127.5\left(\mathrm{C}_{\mathrm{Ar}}\right)\right], 85.4(\mathrm{C}), 57.9(\mathrm{CCH}), 43.0\left(\mathrm{CCH}_{2}\right), 38.6$ $\left(\mathrm{CCH}_{2}\right), 33.4\left(\mathrm{CCH}_{2}\right), 27.7(\mathrm{CCH}), 25.3\left(\mathrm{CCH}_{3}\right), 25.2\left(\mathrm{CCH}_{2}\right)$, $19.1\left(\mathrm{CCH}_{3}\right), 8.1\left(\mathrm{CCH}_{3}\right) \mathrm{ppm}$; HR-MS (ESI, $\left.\mathrm{m} / z\right)$ : calcd. for $\mathrm{C}_{19} \mathrm{H}_{25} \mathrm{NO}_{4}\left[M+\mathrm{NH}_{4}\right]^{+}: 349.2122$, found: 349.2128 .

1-((R)-1-Phenylethyl)-3-(phenylsulfonyl)piperidine-2,6-dione (6, $\left.\mathrm{C}_{19} \mathrm{H}_{19} \mathrm{NO}_{4} \mathrm{~S}\right)$. Yield 68\%; ${ }^{1} \mathrm{H}$ NMR $\left(200 \mathrm{MHz}, \mathrm{CDCl}_{3}\right)$ : $\delta=7.90-7.26(\mathrm{~m}, 10 \mathrm{H}, \mathrm{Ar}-\mathrm{H}), 6.19-6.00(\mathrm{~m}, 1 \mathrm{H}, \mathrm{CH}), 4.13-$ $3.942(\mathrm{~m}, 1 \mathrm{H}, \mathrm{CH}), 3.44-3.14\left(\mathrm{~m}, 1 \mathrm{H}, \mathrm{CH}_{2}\right), 2.92-2.71(\mathrm{~m}, 2 \mathrm{H}$, $\left.\mathrm{CH}_{2}\right), 2.45-2.25\left(\mathrm{~m}, 1 \mathrm{H}, \mathrm{CH}_{2}\right), 1.77-1.71\left(\mathrm{~m}, 3 \mathrm{H}, \mathrm{CH}_{3}\right) \mathrm{ppm} ;{ }^{13} \mathrm{C}$ NMR $\left(50 \mathrm{MHz}, \mathrm{CDCl}_{3}\right): \delta=171.1\left(\mathrm{CCO}_{\text {imide }}\right), 170.8\left(\mathrm{CCO}_{\text {imide }}\right)$, $[139.6,134.5,134.4,129.2,129.0,128.1,128.0,127.7,127.0$, $\left.126.8\left(\mathrm{C}_{\mathrm{Ar}}\right)\right], 66.2(\mathrm{CCH}), 50.3(\mathrm{CCH}), 50.1(\mathrm{CCH}), 29.8\left(\mathrm{CCH}_{2}\right)$, $29.4\left(\mathrm{CCH}_{2}\right), 17.6\left(\mathrm{CCH}_{3}\right), 17.4,\left(\mathrm{CCH}_{3}\right), 15.8\left(\mathrm{CCH}_{2}\right) \mathrm{ppm}$; HRMS (ESI, $m / z)$ : calcd. for $\mathrm{C}_{19} \mathrm{H}_{19} \mathrm{NO}_{4} \mathrm{~S}[M+\mathrm{H}]^{+}$: 358.1099, found: 358.1108 .

2-Benzyl-2-azaspiro[5.11]heptadecane-1,3,7-trione (7, $\mathrm{C}_{23} \mathrm{H}_{31} \mathrm{NO}_{3}$ ). Yield 42.1\%; ${ }^{1} \mathrm{H} \mathrm{NMR}\left(500 \mathrm{MHz}, \mathrm{CDCl}_{3}\right)$ : $\delta=7.34-7.23 \quad(\mathrm{~m}, \quad 5 \mathrm{H}, \quad \mathrm{Ar}-\mathrm{H}), \quad 4.95 \quad\left(\mathrm{AB}_{\mathrm{q}}, \quad 2 \mathrm{H}, \quad \mathrm{CH}_{2}\right.$, $J=14.6 \mathrm{~Hz}), 3.23-3.17\left(\mathrm{ddd}, 1 \mathrm{H}, \mathrm{CH}_{2}, J=7.6 \mathrm{~Hz}, J=5.3 \mathrm{~Hz}\right.$, $J=1.0 \mathrm{~Hz}), 2.68-2.56\left(\mathrm{~m}, 2 \mathrm{H}, \mathrm{CH}_{2}\right), 2.59-2.53\left(\mathrm{~m}, 2 \mathrm{H}, \mathrm{CH}_{2}\right)$, 2.00-1.95 (m, 1H, $\left.\mathrm{CH}_{2}\right), 1.60-1.47$ (m, 4H, $\left.\mathrm{CH}_{2}\right), 1.33-1.19$ (m, $\left.15 \mathrm{H}, \mathrm{CH}_{2}\right), 0.96-0.89\left(\mathrm{~m}, 1 \mathrm{H}, \mathrm{CH}_{2}\right) \mathrm{ppm} ;{ }^{13} \mathrm{C}$ NMR $(125 \mathrm{MHz}$, $\left.\mathrm{CDCl}_{3}\right): \quad \delta=204.8 \quad\left(\mathrm{CCO}_{\text {keto }}\right), \quad 172.7 \quad\left(\mathrm{CCO}_{\text {imide }}\right), \quad 171.9$ $\left(\mathrm{CCO}_{\text {imide }}\right),\left[136.9,128.8,128.4,127.5\left(\mathrm{C}_{\mathrm{Ar}}\right)\right], 60.0(\mathrm{CCH})$, $43.4\left(\mathrm{CCH}_{2}\right), 34.7\left(\mathrm{CCH}_{2}\right), 33.9\left(\mathrm{CCH}_{2}\right), 29.9\left(\mathrm{CCH}_{2}\right), 26.3$ $\left(\mathrm{CCH}_{2}\right), 26.2\left(\mathrm{CCH}_{2}\right), 23.3\left(\mathrm{CCH}_{2}\right), 23.1\left(\mathrm{CCH}_{2}\right), 22.0\left(\mathrm{CCH}_{2}\right)$, $21.7\left(\mathrm{CCH}_{2}\right), 18.8\left(\mathrm{CCH}_{2}\right)$ ppm; HR-MS (ESI, $\left.\mathrm{m} / z\right)$ : calcd. for $\mathrm{C}_{23} \mathrm{H}_{31} \mathrm{NO}_{3}[M+\mathrm{H}]^{+}:$370.2377, found: 370.2371.
2-Benzyl-2-azaspiro[5.7]tridecane-1,3,7-trione $\left(\mathbf{8}, \mathrm{C}_{19} \mathrm{H}_{23} \mathrm{NO}_{3}\right)$. Yield 53\%; ${ }^{1} \mathrm{H}$ NMR $\left(200 \mathrm{MHz}, \mathrm{CDCl}_{3}\right): \delta=7.28-7.23(\mathrm{~m}, 5 \mathrm{H}$, $\mathrm{Ar}-\mathrm{H}), 4.91$ (s, 2H, CH ), 3.19-2.40 (m, 6H, $\left.\mathrm{CH}_{2}\right), 2.25$ (ddd, $\left.J=12.3 \mathrm{~Hz}, J=6.2 \mathrm{~Hz}, J=3.4 \mathrm{~Hz}, 1 \mathrm{H}, \mathrm{CH}_{2}\right), 1.88-1.53(\mathrm{~m}, 9 \mathrm{H}$, $\left.\mathrm{CH}_{2}\right) \mathrm{ppm} ;{ }^{13} \mathrm{C} \mathrm{NMR}\left(50 \mathrm{MHz}, \mathrm{CDCl}_{3}\right): \delta=213.3\left(\mathrm{CCO}_{\mathrm{keto}}\right)$, $172.3\left(\mathrm{CCO}_{\text {imide }}\right), 171.6\left(\mathrm{CCO}_{\text {imide }}\right),[137.0,128.4,128.2,127.3$ $\left(\mathrm{C}_{\mathrm{Ar}}\right)$ ], $57.9(\mathrm{C}), 43.1\left(\mathrm{CCH}_{2}\right), 38.3\left(\mathrm{CCH}_{2}\right), 31.4\left(\mathrm{CCH}_{2}\right), 30.0$ $\left(\mathrm{CCH}_{2}\right), 29.5\left(\mathrm{CCH}_{2}\right), 25.7\left(\mathrm{CCH}_{2}\right), 23.9\left(\mathrm{CCH}_{2}\right), 23.8\left(\mathrm{CCH}_{2}\right)$, $22.5\left(\mathrm{CCH}_{2}\right)$ ppm; HR-MS (ESI, m/z): calcd. for $\mathrm{C}_{19} \mathrm{H}_{23} \mathrm{NO}_{3}$ $[M+\mathrm{H}]^{+}:$314.1751, found: 314.1738 .

Ethyl 4-(1-benzyl-2,6-dioxopiperidin-3-yl)butanoate (9, $\left.\mathrm{C}_{18} \mathrm{H}_{23} \mathrm{NO}_{4}\right)$. Yield 42\%; ${ }^{1} \mathrm{H}$ NMR $\left(200 \mathrm{MHz}, \mathrm{CDCl}_{3}\right)$ : $\delta=7.37-7.22(\mathrm{~m}, 5 \mathrm{H}, \mathrm{Ar}-\mathrm{H}), 4.94\left(\mathrm{~s}, 2 \mathrm{H}, \mathrm{CH}_{2}\right), 4.13(\mathrm{q}$, $\left.J=7.1 \mathrm{~Hz}, 2 \mathrm{H}, \mathrm{CH}_{2}\right), 2.82\left(\mathrm{dt}, J=17.6 \mathrm{~Hz}, J=4.9 \mathrm{~Hz}, 1 \mathrm{H}, \mathrm{CH}_{2}\right)$, $2.64\left(\mathrm{dd}, J=11.1 \mathrm{~Hz}, J=5.3 \mathrm{~Hz}, 1 \mathrm{H}, \mathrm{CH}_{2}\right), 2.54-2.42(\mathrm{~m}, 1 \mathrm{H}$, $\mathrm{CH}), 2.34\left(\mathrm{t}, J=7.2 \mathrm{~Hz}, 2 \mathrm{H}, \mathrm{CH}_{2}\right), 2.12-1.90\left(\mathrm{~m}, 2 \mathrm{H}, \mathrm{CH}_{2}\right)$, $1.82-1.56\left(\mathrm{~m}, 4 \mathrm{H}, \mathrm{CH}_{2}\right), 1.25\left(\mathrm{t}, J=7.1 \mathrm{~Hz}, 3 \mathrm{H}, \mathrm{CH}_{3}\right) \mathrm{ppm} ;{ }^{13} \mathrm{C}$ $\operatorname{NMR}\left(50 \mathrm{MHz}, \mathrm{CDCl}_{3}\right): \delta=174.5\left(\mathrm{CCO}_{\text {imide }}\right), 173.2\left(\mathrm{CCO}_{\text {ester }}\right)$, $172.2\left(\mathrm{CCO}_{\text {imide }}\right),\left[137.3,128.5,128.3,127.3\left(\mathrm{C}_{\mathrm{Ar}}\right)\right], 60.3$ $\left(\mathrm{CCH}_{2}\right), 42.9\left(\mathrm{CCH}_{2}\right), 41.9\left(\mathrm{CCH}_{2}\right), 33.9\left(\mathrm{CCH}_{2}\right), 31.9\left(\mathrm{CCH}_{2}\right)$, $29.6\left(\mathrm{CCH}_{2}\right), 22.2\left(\mathrm{CCH}_{2}\right), 22.0\left(\mathrm{CCH}_{2}\right), 14.1\left(\mathrm{CCH}_{3}\right) \mathrm{ppm}$; HR-MS (ESI, $m / z)$ : calcd. for $\mathrm{C}_{18} \mathrm{H}_{23} \mathrm{NO}_{4}[M+\mathrm{H}]^{+}$: 318.1700, found: 318.1691.

8-Oxaspiro[4.5]decane-7,9-dione (2c) was prepared according to a modified literature procedure ${ }^{47}$.

7,9-Dioxo-8-azaspiro[4.5]decane-6,10-dicarbonitrile (2a) and 2,2'-cyclopentane-1,1-diyldi acetic acid (2b) were prepared by a known literature procedure ${ }^{48}$. Methyl 5-chloro-5-oxopentanoate (1b) and methyl [1-(2-chloro-2-oxoethyl)cyclopentyl] acetate (2d) were prepared by a modification of a known literature procedure $^{49}$.

Methyl 5-(benzylamino)-5-oxopentanoate (1c) and methyl 2-(1-(2(benzylamino)-2-oxoethyl) cyclopentyl) acetate (2e) were prepared according to a modified literature procedure ${ }^{50}$.

Methyl 2-methylbutan-2-yl propanedioate $(\mathbf{3 b})$ was prepared by a literature procedure ${ }^{51}$. Methyl 2-(phenylsulfonyl)acetate (3c) was prepared by a literature procedure ${ }^{52}$.

Acrylamides (4a, 4c, 4d) and (E)-N-benzylbut-2-enamide (4b) were prepared according to a modified literature procedure ${ }^{50}$.

$\beta$-Ketoesters $(\mathbf{5 a}-\mathbf{c})$ were prepared by a modification of a literature procedure ${ }^{53}$.

\section{Results and discussion}

\section{Chemistry}

Glutarimide derivatives, depicted in Figure 1, were synthesized according to previously reported procedures of our $^{30}$ or other research groups. Compounds $\mathbf{1}$ and $\mathbf{2}$ were prepared according to a modified literature procedure. Cyclization of amido-esters, derived from corresponding glutaric acid anhydrides, in presence of the base $(\mathrm{NaH})$, was carried out by reflux in toluene $(\sim 3 \mathrm{~h})$, yielding glutarimides $\mathbf{1}$ and $\mathbf{2}$ (Scheme 1). Amido-esters $\mathbf{1 c}$ and $\mathbf{2 e}$ were prepared from $\mathbf{1 a}$ and $\mathbf{2 c}$, respectively, by a modification of standard methods ${ }^{47,49,50}$.

Compounds 3-9 were synthesized by tandem process described in our previous paper ${ }^{30}$. The process involved a base-catalyzed Michael addition of active methylene compounds to secondary acrylamides or crotonamides, followed by intramolecular $\mathrm{N}$-acylation of the carboxamido group. Synthesis of derivatives 3-6 was performed by reacting methyl 1,2-cyanoacetate (3a), methyl $t$-pentyl malonate (3b) and methyl 2-(phenylsulfonyl)acetate $(\mathbf{3 c})$ with $N$-substituted acryl- and crotonamides $(\mathbf{4 a - c})$, under the reaction conditions (Scheme 2A). Yields of products were $42-72 \%$. In the reaction of $\beta$-keto esters, comprising 5, 8 and 12 member rings, 5a-c, with $N$-benzyl acrylamide, imides $\mathbf{9 , 8}$ and 7, respectively, were obtained (Scheme $2 \mathrm{~B}$ ). 
Scheme 1. Synthetic path to obtain compounds $\mathbf{1}$ and $\mathbf{2}$.

Scheme 2. Synthetic paths to obtain compounds (A) 3-6; and (B) 7-9.
(A)

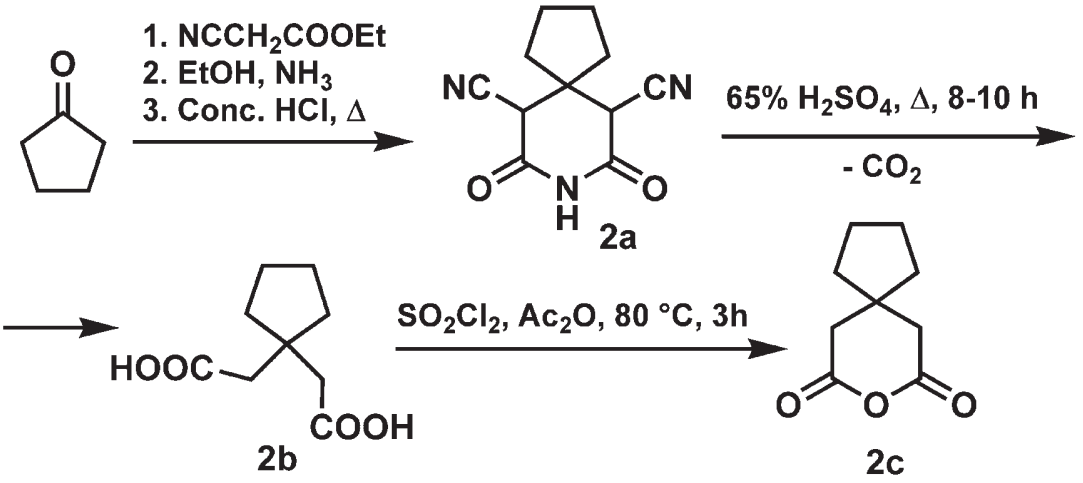

(B)

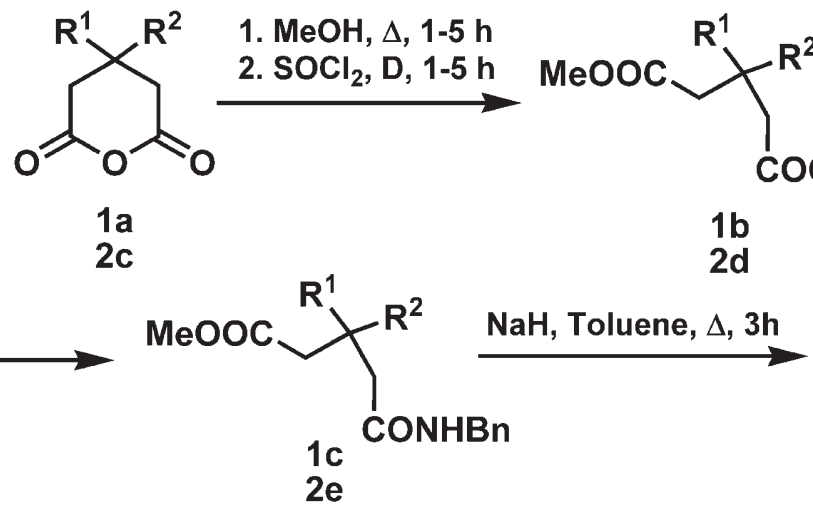

1a, 1b, 1c, 1: $R^{1}, R^{2}=H$

2c, 2d, 2e, 2: $\mathbf{R}^{1}, \mathbf{R}^{2}=-\mathrm{CH}_{2} \mathrm{CH}_{2} \mathrm{CH}_{2} \mathrm{CH}_{2-}$

(A)<smiles>[X]CC(=O)OC</smiles>
3a-c

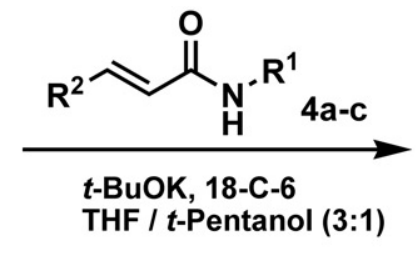

4a; $\mathbf{R}^{1}=$ 2-Phen; $\mathbf{R}^{2}=\mathrm{H}$

$4 b ; R^{1}=B n ; R^{2}=M e$

$4 c ; R^{1}=(S)-2-P h e n ; R^{2}=H$<smiles>[R]C1CC(=O)N([R1])C(=O)C1[X]</smiles>

$3 a ; X=C N$

$3 \mathrm{~b} ; \mathrm{X}=\mathrm{COO}-\mathrm{t}$-Pent

$3 \mathrm{c} ; \mathrm{X}=\mathrm{SO}_{2} \mathrm{Ph}$

3; $X=C N ; R^{1}=2-P h e n ; R^{2}=~ H$

4; $X$ = COO-t-Pent; $\mathbf{R}^{1}=$ 2-Phen; $\mathbf{R}^{2}=\mathrm{H}$

5; $X$ = COO-t-Pent; $\mathbf{R}^{1}=\mathrm{Bn} ; \mathrm{R}^{2}=\mathrm{Me}$

6; $\mathrm{X}=\mathrm{SO}_{2} \mathrm{Ph} ; \mathrm{R}^{1}=(\mathrm{S})-2-\mathrm{Phen} ; \mathrm{R}^{2}=\mathrm{H}$

(B)<smiles>CC(C)C1CCC(=O)C(C(=O)O)C1</smiles>

5a-c

$5 \mathrm{a} ; \mathrm{n}=3 ; \mathrm{R}=\mathrm{Me}$

$5 b ; n=7 ; R=M e$

$5 c ; n=0 ; R=E t$<smiles>CCOC(=O)CCCC1CCC(=O)N(Cc2ccccc2)C1=O</smiles> 


\section{Antiproliferative activity}

The cytotoxicity of compounds 1-9 was tested toward selected human cancer cell lines: cervix adenocarcinoma HeLa, human myelogenous leukemia K562 and human breast carcinoma MDAMB-453 cells. Cell survival was determined by MTT test, after

Table 1. Concentrations of compounds 1-9 that induced 50\% decrease in cell survival $\left(\mathrm{IC}_{50}\right)$.

\begin{tabular}{lcccc}
\hline & \multicolumn{4}{c}{$\mathrm{IC}_{50}(\mu \mathrm{M})$} \\
\cline { 2 - 5 } Compound & HeLa & K562 & MDA-MB-453 & MRC5 \\
\hline $\mathbf{1}$ & $>200$ & $>200$ & $>200$ & - \\
$\mathbf{2}$ & $150.2 \pm 2.0$ & $103.9 \pm 4.4$ & $167.8 \pm 3.8$ & - \\
$\mathbf{3}$ & $>200$ & $>200$ & $>200$ & - \\
$\mathbf{4}$ & $119.3 \pm 1.3$ & $96.6 \pm 2.5$ & $150.9 \pm 0.2$ & - \\
$\mathbf{5}$ & $108.4 \pm 8.1$ & $81.1 \pm 0.8$ & $135.7 \pm 0.2$ & - \\
$\mathbf{6}$ & $154.3 \pm 6.9$ & $147.1 \pm 8.7$ & $\sim 200$ & - \\
$\mathbf{7}$ & $26.8 \pm 4.7$ & $8.98 \pm 0.64$ & $27.36 \pm 0.20$ & $36.73 \pm 0.65$ \\
$\mathbf{8}$ & $146.7 \pm 5.9$ & $150.0 \pm 0.1$ & $138.1 \pm 3.5$ & - \\
$\mathbf{9}$ & $186.5 \pm 1.2$ & $193.6 \pm 6.4$ & $195.2 \pm 4.8$ & - \\
\hline
\end{tabular}

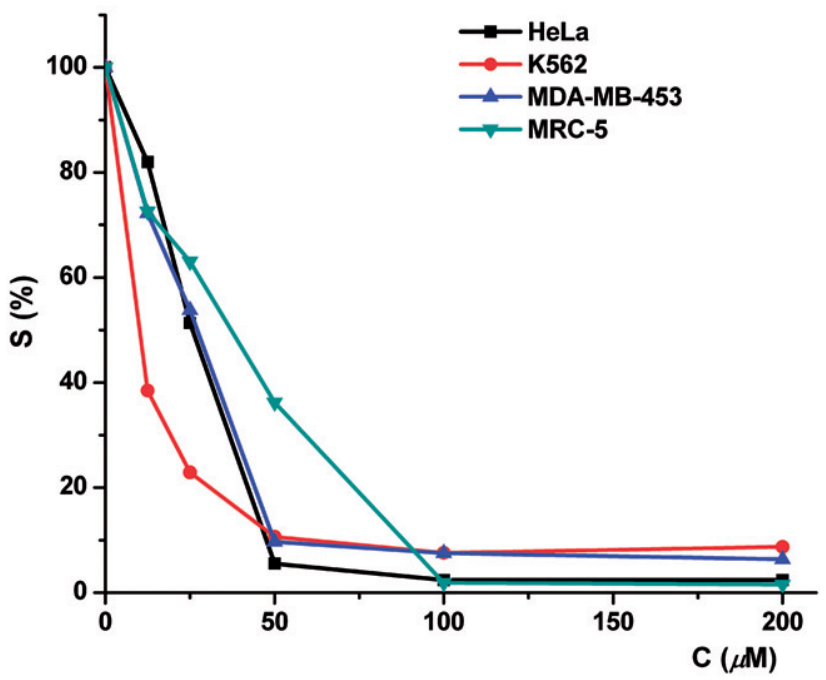

Figure 2. Dose-response curves for the cytotoxicity of compound 7 toward HeLa, K562, MDA-MB-453 and MRC-5 cells. Percentage of viable cells $(\mathrm{S} \%)$ was plotted against various concentrations of compound 7.
$72 \mathrm{~h}$ of exposure to compounds ${ }^{31,32} . \mathrm{IC}_{50}$ values (Table 1) are shown in molar concentrations, as the mean $\pm \mathrm{SD}$, determined from three independent measurements.

$\mathrm{IC}_{50}$ was defined as the concentration of the compound inhibiting cell survival by $50 \%$, compared with a vehicle-treated control cells. The most potent compound, 7, was also tested toward MRC-5, normal lung fibroblast cells. Compounds 2, 4, 5$\mathbf{8}$ and $\mathbf{9}$ exerted dose-dependent cytotoxicity toward malignant cells, while compound 7 appeared as the most active. Compound 7 exerted humble selectivity, with $\mathrm{IC}_{50}$ toward normal cells $\sim 37 \mu \mathrm{M}$. The decrease in survival of target cells induced by compound 7 is shown in Figure 2.

\section{Antibacterial activity}

Antibacterial activity of compounds 1, 2, 4-8 and 9 was examined against selected foodborne pathogenic bacteria, Gram-positive species S. aureus (ATCC 25923), E. faecalis (ATCC 29212), B. cereus (ATCC 10876), L. monocytogenes (ATCC 19115) and Gram-negative species $P$. aeruginosa (ATCC 27853), E. coli (ATCC 25922), S. enteritidis (ATCC 13076), P. hauseri (ATCC 13315), S. sonnei (ATCC 29930), Y. enterocolitica (ATCC 27729), E. coli O157:H7 (ATCC 12900). A preliminary screening, done by a disc diffusion method, indicated the ability of bacteria to produce visible growth in the presence of compounds $\mathbf{1 ,}, \mathbf{2}, \mathbf{4}-\mathbf{8}$ and 9. In most cases, Gram-negative bacteria were more resistant to the tested compounds than Gram-positive bacteria (Table 2). The diameters of the inhibition zones, determined by a disk diffusion method, ranged from 6.4 to $14.6 \mathrm{~mm}$, in the presence of $200 \mu \mathrm{g}$ of compounds tested.

The Gram-positive bacteria $B$. cereus were the most sensitive to compounds 4 and 9 (14.6 and $14.2 \mathrm{~mm}$, respectively). Such activity was comparable to the effect of commercial antibiotic gentamicin $(14.8 \mathrm{~mm})$. Also, there was no statistically significant difference between the susceptibility of $B$. cereus on commercial antibiotic tetracycline (12.4 $\mathrm{mm}$ ) and compounds $\mathbf{2}, \mathbf{7}$ and $\mathbf{8}$ (12.1, 12.9 and $12.4 \mathrm{~mm}$, respectively). Among Gram-negative bacteria, $S$. sonnei was the most sensitive to compounds 2,4 and $\mathbf{6}$ (11.5, 10.4 and $10.2 \mathrm{~mm}$, respectively), comparable to antibiotic tetracycline $(11.4 \mathrm{~mm})$. Other compounds did not show significant antimicrobial activity against tested bacteria in the applied concentration. Compound $\mathbf{5}$ did not show any antimicrobial effect on tested Gram-positive and Gram-negative bacteria.

Due to the fact that the disk diffusion method is not entirely reliable in determining the antimicrobial properties, the broth microdilution method, as a rapid and quantitative method

Table 2. Antibacterial activity of compounds $\mathbf{1}, \mathbf{2}, \mathbf{4}, \mathbf{6}-\mathbf{8}$ and $\mathbf{9}$, determined by the disk diffusion method.

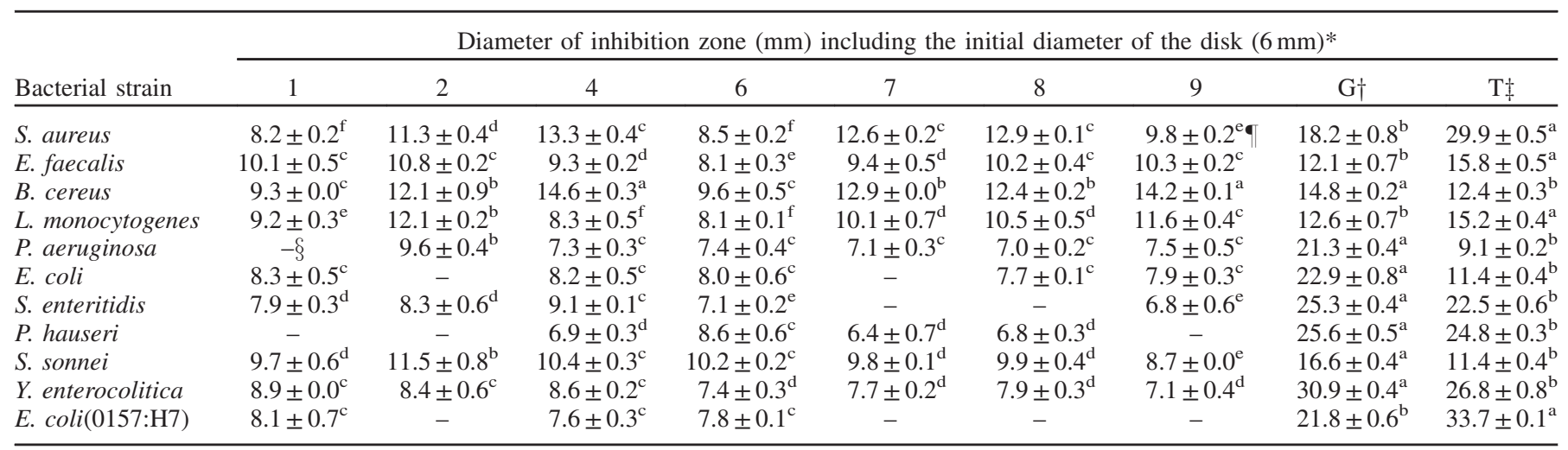

*Data are expressed as mean \pm standard deviation $(n=3)$.

$\dagger$ G-gentamicin.

$\ddagger \mathrm{T}$-tetracycline

TWithin the same row, means followed by different letters are significantly different at $p<0.05$ (ANOVA, Fisher's LSD).

$\S$ Inhibition zone not achieved. 


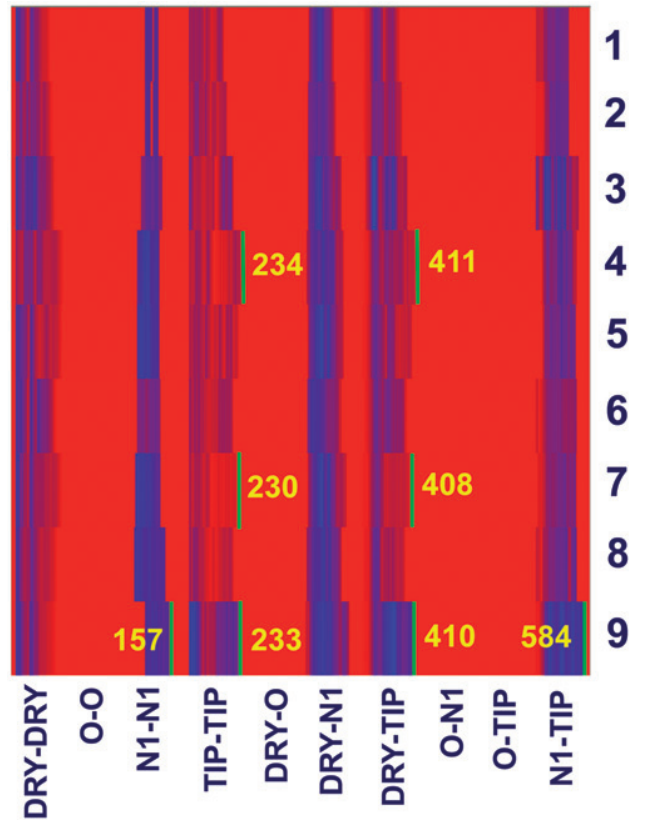

Figure 3. Heatmap depiction of descriptors (auto- and cross-correlograms), calculated by AMANDA algorithm, for compounds 1-9.

for determining of MIC, based on the color change caused by the enzymatic activity of viable microorganisms, was applied (Table 3). Compounds 1, 2, 4, 6-8 and 9 inhibited the growth of all tested Gram-positive and some of the Gram-negative bacteria. Achieved MICs were in the range of $0.625-10.0 \mathrm{mg} / \mathrm{mL}$ $\left(1.97 \times 10^{-3}-4.92 \times 10^{-2} \mathrm{~mol} / \mathrm{L}\right)$.

The highest antibacterial potential was reached with compound 9 against $B$. cereus (MIC was $0.625 \mathrm{mg} / \mathrm{mL}=1.97 \times 10^{-3} \mathrm{~mol} / \mathrm{L}$ ). MICs against Gram-negative bacteria exceeded $10.0 \mathrm{mg} / \mathrm{mL}$, for the majority of compounds, including compound $\mathbf{5}$, proved to be inactive in concentrations up to $10.0 \mathrm{mg} / \mathrm{mL}$ against all bacterial strains tested. Exceptions were compound 4, whose antibacterial activity ranged from 5.0 to $10.0 \mathrm{mg} / \mathrm{mL}$ against all tested Gramnegative bacteria and compounds 2,6 and 7 , with MICs of $10.0 \mathrm{mg} / \mathrm{mL}\left(3.89 \times 10^{-2}, 2.80 \times 10^{-2}\right.$ and $2.71 \times 10^{-2} \mathrm{~mol} / \mathrm{L}$, respectively) against $S$. sonnei.

\section{Structure-activity relationship}

Although exerting humble potency, derivatives $\mathbf{9}$ and $\mathbf{4}$ appeared most potent against bacteria, while derivative 7 was the one with fair potency toward human tumor cells. In order to rationalize structural features associated with the most potent compounds we calculated molecular descriptors derived from the 3D structures of compounds. For modeling studies structures of all compounds (1-9) were prepared as described in our previous paper ${ }^{54}$, see section "Methods". Due to low number of compounds tested against bacteria, or toward human tumor cells, and limited potencies, we did not build novel quantitative structure-activity models, but envisaged to use molecular properties and to use previously built model to draw conclusions on structural characteristics needed for significant potency. To predict potency of compound 7 toward K562 cells, we projected structures of compounds 2, 7 and $\mathbf{8}$ (all spirocyclic compounds in our dataset) on model previously derived for antiproliferative activity data of glutarimide derivatives toward K562 cells, collected from National Cancer Institute (NCI) repository ${ }^{54}$. Unfortunately, our prediction failed. Compounds $\mathbf{2}$ and $\mathbf{7}$ were predicted as more potent comparing to compound $\mathbf{8}$ (data not shown). There are several possible reasons for the poor prediction. First, in the

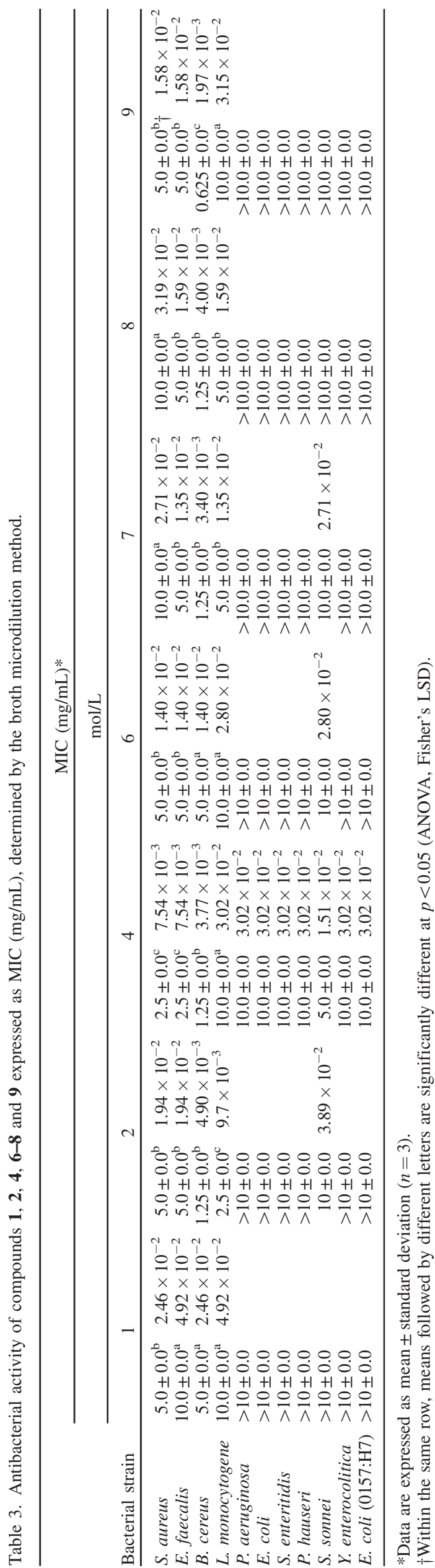


model of antiproliferative activity of NCI glutarimides toward $\mathrm{K} 562$ cells, potency data span range from 4 to $8.6\left(\mathrm{p}\left(\mathrm{GI}_{50}\right)\right.$ values). Approximating equality between $\mathrm{p}\left(\mathrm{IC}_{50}\right)$ and $\mathrm{p}\left(\mathrm{GI}_{50}\right)$ values, which for sure is not the most stringent criteria; compounds 2 and 7, with $\mathrm{p}\left(\mathrm{IC}_{50}\right)$ of 3.98 and 3.82, respectively, are out of the range of potencies that NCI model covers. Next, antiproliferative data of compounds tested by NCI were obtained by Sulforhodamine B assay after $48 \mathrm{~h}$ of exposure of cells to compounds, while we used MTT assay and exposed cells to compounds during $72 \mathrm{~h}$. The most potent compounds in NCI set comprise unsubstituted glutarimide nitrogen, while all compounds in our set bear bulky substituent in this position. Finally, our model of antiproliferative activity of NCI glutarimides was built on the pharmacophoric similarity patterns (including spatial positions of HBA, HBD, hydrophobic and shape features), but did not include whole-molecule properties, as surfaces and volumes. Next, we compared whole-molecular properties of compounds 19 (surface area, polar surface area, apolar surface area, volume, virtual $\log P$; Supplemental Information, Table S1) derived from the $3 \mathrm{D}$ structures of compounds. We concluded that derivative 7 , most potent toward human tumor cells tested, had the largest molecular volume. This observation is in accordance with the fact that compounds having largest molecular volumes are among most potent in NCI glutarimide set (Figure $4 \mathrm{a}$ in reference ${ }^{54}$ ). To obtain more data on structural features associated with most potent compounds in our set, we calculated MIF, with hydrogenbond donor (N1), hydrogen-bond acceptor (O), hydrophobic (DRY) and shape (TIP) probes around compounds 1-9 in program Pentacle, and visually inspected interaction patterns, obtained by two-point pharmacophoric features offered by AMANDA algorithm $^{45,55}$. Blocks of correlograms were depicted as a heatmap (Figure 3). Features that separate the most potent compounds from the rest appeared straightforwardly.

We observed TIP-TIP and DRY-TIP blocks of variables which were broader for compounds $\mathbf{4 , 7}$ and $\mathbf{9}$, comparing with the same block of variables for the rest of compounds. Along with this, the N1-TIP block of variables is significantly broader for compound 9, compared to other compounds. So, variables in those three blocks, with the largest distances between nodes, made distinction between compounds $\mathbf{4}, 7$ and 9 and the rest. Variables TIP-TIP230 , encoding nodes of shape probe on distance of $\sim 17.1 \AA$ (Supplemental Information, Figure S19a), and DRY-TIP-408, encoding node of hydrophobic probe and the node of shape probe on distance of $\sim 17.4 \AA$ (Supplemental Information, Figure S19b), clearly separated compound 7, most potent toward tumor cells tested, from the rest. Along the largest molecular volume, compound 7 comprises bulky cycloalkyl moiety distal from the benzyl ring bound to glutarimide $\mathrm{N}$. We observed similar structural features for compounds 9 and 4, most active against Gram-positive bacteria. Variables TIP-TIP-233 (nodes of the shape probe on distance of $\sim 18.1 \AA$ ) and DRY-TIP-410 (node of the hydrophobic probe on distance of $\sim 18.1 \AA$ from the node of shape probe) encoded compound 9 . Variables TIP-TIP-234 (nodes of the shape probe on distance of $\sim 18.4 \AA$ ) and DRY-TIP-411 (node of the hydrophobic probe on distance of $\sim 18.4 \AA$ from the node of shape probe) encode compound 4. Along with those two variables, comparable with variables associated with compound $\mathbf{7}$, that described overall shape and spatial position of benzyl moiety and branched alkyl chains attached to position 3 of glutarimide ring in compounds 9 and 4; we observed additional variables characteristic for compound 9. Variables N1-TIP-584 (HBD associated with keto group in glutarimide ring, and alkyl moiety of the ester, associated with the shape probe, on spatial distance of $\sim 17.1 \AA$ ) and N1-N1-157 (HBDs associated with keto group in glutarimide ring and with ester keto moiety) are typical for compound 9. We did not observe similar variables for compound
4, most probably because ester keto group, as a HBA, was hindered with branched alkyl moiety. All variables described for compounds 9 and $\mathbf{4}$ are depicted in Figures S20 and S21 (Supplemental Information). So, along with overall large molecular volumes, due to alkyl chains bound to position 3 of glutarimide moiety, compound 9, which exerted better antibacterial activity than the rest of compounds, has HBA moiety in this alkyl chain and this HBA is not hindered by the vicinal parts of molecule.

\section{Conclusion}

The cytotoxicity study of compounds 1-9 toward selected human cancer cell lines showed that compound 7 was the most potent, with $\mathrm{IC}_{50}$ values 8.98, 26.8 and $27.36 \mu \mathrm{M}$, toward K562, HeLa and MDA-MB-453 cells, respectively. This compound exerted modest selectivity toward normal control MRC-5 cells with $\mathrm{IC}_{50} \sim 37 \mu \mathrm{M}$. Preliminary screening of antibacterial activity by a disk diffusion method showed that Gram-positive bacteria were more susceptible to tested compounds than Gram-negative bacteria. Compounds 4 and $\mathbf{9}$ expressed the highest inhibition on growth of Gram-positive bacteria $B$. cereus and it is comparable to antibiotic gentamicin. $S$. sonnei (Gram-negative bacteria) was the most sensitive to the compound 2 (comparable to antibiotic tetracycline). Using a quantitative method for determination of MIC the highest antibacterial potential was achieved with compound 9 against B. cereus. Compound $\mathbf{5}$ did not show any antimicrobial effect on tested Gram-positive and Gram-negative bacteria in any of applied tests. Whole-molecular descriptors derived from 3D structures of examined compounds and structure-activity model based on MIF, calculated by the GRID method, rationalized structural features associated with the most potent compounds. Compound with bulky hydrophobic moiety distal from glutarimide ring exerted best antiproliferative potency within studied set.

We found that compound 7 exerts the highest antiproliferative potency within studied set. This derivative will be a starting point for further structural modifications in order to obtain more potent compounds. As compound 7 exerts modest selectivity, the second goal of structural modifications will be improvement of selectivity of new derivatives.

\section{Declaration of interest}

The authors have no conflicts of interest. Authors acknowledge the Ministry of Education, Science and Technological Development of the Republic of Serbia for financial support (Grant Nos. 172032 and 175011).

\section{References}

1. Matsuda F, Terashima S. Total synthesis of natural (+)-sesbanimide A and (-)-sesbanimide B. Tetrahedron 1988;44:4721-36.

2. Ju J, Rajski SR, Lim SK, et al. Lactimidomycin iso-migrastatin and related glutarimide-containing 12-membered macrolides are extremely potent inhibitors of cell migration. J Am Chem Soc 2009;131: 1370-1.

3. Schneider-Poetsch T, Ju J, Eyler DE, et al. Inhibition of eukaryotic translation elongation by cycloheximide and lactimidomycin. Nat Chem Biol 2010;6:209-17.

4. Sugawara K, Nishiyama Y, Toda S, et al. Lactimidomycin, a new glutarimide group antibiotic. Production, isolation, structure and biological activity. J Antibiot 1992;45:1433-41.

5. Machado AL, Lima LM, Araujo Jr JX, et al. Design, synthesis and antiinflammatory activity of novel phthalimide derivatives, structurally related to thalidomide. Bioorg Med Chem Lett 2005;15: 1169-72.

6. Bartlett JB, Dredge K, Dalgleish AG. The evolution of thalidomide and its IMiD derivatives as anticancer agents. Nat Rev Cancer 2004; 4:314-22. 
7. Wu YH, Rayburn JW, Allen LE, et al. Psychosedative agents. 2. 8(4-Substituted 1-piperazinylalkyl)-8-azaspiro[4.5]decane-7,9-diones. J Med Chem 1972;15:477-9.

8. Barrdell LB, Fitton A. Tandospirone. CNS Drugs 1996;5:147-52.

9. Obrig TG, Culp WJ, Mckeehan WL, Hardesty B. The mechanism by which cycloheximide and related glutarimide antibiotics inhibit peptide synthesis on reticulocyte ribosomes. J Biol Chem 1971;246: 174-81.

10. Kondo H, Oritani T, Kiyota H. Synthesis and antifungal activity of the four stereoisomers of streptimidone, a glutarimide antibiotic from Streptomyces rimosus forma paromomycinus. Eur J Org Chem 2000;2000:3459-62.

11. Frohardt RP, Dion HW, Jakubowski ZL, et al. Chemistry of streptimidone, a new antibiotic. J Am Chem Soc 1959;81:5500-6.

12. Kim BS, Moon SS, Hwang BK. Isolation, antifungal activity, and structure elucidation of the glutarimide antibiotic, streptimidone, produced by Micromonospora coerulea. J Agric Food Chem 1999; 47:3372-80.

13. Ha DKK, Lau WH. Effect of recombinant human tumor necrosis factor on human nasopharyngeal carcinoma cell line in vitro. Cancer Lett 1988;41:217-24.

14. Andres MI, Sanz P, Garfia A, et al. Induction of cell differentiation and other in vitro effects of cycloheximide on neuroblastoma cells. In Vitro Mol Toxicol 1977;10:319-28.

15. Ishikawa $Y$, Tachibana $M$, Matsui $C$, et al. Synthesis and biological evaluation on novel analogs of 9-methylstreptimidone, an inhibitor of NF-кB. Bioorg Med Chem Lett 2009;19:1726-8.

16. Powell RG, Smith Jr CR, Weisleder D. Sesbanimide A and related tumor inhibitors from Sesbania drummondii: structure and chemistry. Phytochemistry 1984;23:2789-96.

17. Powell RG, Smith CR, Weisleder D, et al. Sesbanimide, a potent antitumor substance from Sesbania drummondii seed. J Am Chem Soc 1983;105:3739-41.

18. Powel RG, Smith Jr CR. An investigation of the antitumor activity of Sesbania drummondii. J Nat Prod 1981;44:86-90.

19. Powel RG, Smith Jr CR, Madrigal RV. Antitumor activity of Sesbania vesicaria, $S$. punicea, and $S$. drummondii seed extracts. Planta Med 1976;30:1-8.

20. Armoiry X, Aulagner G, Facon T. Lenalidomide in the treatment of multiple myeloma: a review. J Clin Pharm Ther 2008:33:219-26.

21. Lentzsch S, Rogers MS, LeBlanc R, et al. S-3-Amino-phthalimidoglutarimide inhibits angiogenesis and growth of B-cell neoplasias in mice. Cancer Res 2002;62:2300-5.

22. McCarthy PL, Owzar K, Hofmeister CC, et al. Lenalidomide after stem-cell transplantation for multiple myeloma. N Engl J Med 2012; 366:1770-81.

23. Fischer DS, Woo LW, Mahon MF, et al. D-ring modified estrone derivatives as novel potent inhibitors of steroid sulfatase. Bioorg Med Chem 2003;11:1685-700.

24. Brueggemeier RW, Hackett JC, Diaz-Cruz ES. Aromatase inhibitors in the treatment of breast cancer. Endocr Rev 2005;26:331-45.

25. Santen RJ, Brodie H, Simpson ER, et al. History of aromatase: saga of an important biological mediator and therapeutic target. Endocr Rev 2009;30:343-75.

26. Antonini I, Volpini R, Dal Ben D, et al. Design, synthesis, and biological evaluation of new mitonafide derivatives as potential antitumor drugs. Bioorg Med Chem 2008;16:8440-6.

27. Norton JT, Witschi MA, Luong L, et al. Synthesis and anticancer activities of 6-amino amonafide derivatives. Anti-Cancer Drugs 2008; 19:23-36

28. Wu A, Xu Y, Qian X, et al. Novel naphthalimide derivatives as potential apoptosis-inducing agents: design, synthesis and biological evaluation. Eur J Med Chem 2009;44:4674-80.

29. Machado KE, Navakoski de Oliveira K, Santos-Bubniak L, et al. Evaluation of apoptotic effect of cyclic imide derivatives on murine B16F10 melanoma cells. Bioorg Med Chem 2011;19:6285-91.

30. Popović-Đorđević JB, Ivanović MD, Kiricojević VD. A novel tandem process leading to functionalized glutarimides. Tetrahedron Lett 2005;46:2611-14
31. Mosmann T. Rapid colorimetric assay for cellular growth and survival: application to proliferation and cytotoxicity assays. J Immunol Methods 1983;65:55-63.

32. Ohno M, Abe T. Rapid colorimetric assay for the quantification of leukemia inhibitory factor (LIF) and interleukin-6 (IL-6). J Immunol Methods 1991;145:199-203.

33. Tepe B, Daferera D, Sokmen A, et al. Antimicrobial and antioxidant activities of the essential oil and various extracts of Salvia tomentosa Miller (Lamiaceae). Food Chem 2005;90:333-40.

34. Clinical and Laboratory Standards Institute (CLSI). Performance standards for antimicrobial susceptibility testing: 15th informational supplement 2005. CLSI Document M100-S15. Pennsylvania: CLSI.

35. Klančnik A, Piskernik S, Jeršek B, Smole Možina S. Evaluation of diffusion and dilution methods to determine the antibacterial activity of plant extracts. J Microbiol Methods 2010;81:121-6.

36. Sadowski J, Gasteiger J. From atoms and bonds to three-dimensional atomic coordinates: automatic model builders. Chem Rev 1993;93: 2567-81.

37. Sadowski J, Gasteiger J, Klebe G. Comparison of automatic threedimensional model builders using 639 X-ray structures. J Chem Inform Model 1994;34:1000-8.

38. Pedretti A, Villa L,Vistoli G. VEGA - an open platform to develop chemo-bio-informatics applications, using plug-in architecture and script programming. J Comput-Aided Mol Des 2004;18:167-73.

39. Halgren TA. MMFF VI. MMFF94s option for energy minimization studies. J Comput Chem 1999;20:720-9.

40. Harrison RW. Stiffness and energy conservation in molecular dynamics: an improved integrator. J Comput Chem 1993;14: 1112-22.

41. Stewart JJP. Optimization of parameters for semiempirical methods $\mathrm{V}$ : modification of NDDO approximations and application to 70 elements. J Mol Model 2007;13:1173-213.

42. Stewart JJP. MOPAC: a semiempirical molecular orbital program. J Comput-Aided Mol Des 1990;4:1-103.

43. Gaillard P, Carrupt PA, Testa B, Boudon A. Molecular lipophilicity potential, a tool in 3D QSAR: method and applications. J ComputAided Mol Des 1994;8:83-96.

44. Goodford PJ. A Computational procedure for determining energetically favorable binding sites on biologically important macromolecules. J Med Chem 1985;28:849-57.

45. Durán Á, Martínez GC, Pastor M. Development and validation of AMANDA, a new algorithm for selecting highly relevant regions in molecular interaction fields. J Chem Inform Model 2008;48: 1813-23. Pentacle 1.0.6. http://www.moldiscovery.com/software/ pentacle/

46. Gokel GW, Cram DJ, Liotta CL, et al. 18-Crown-6 (1,4,7,10,13,16hexaoxacycloöctadecane). Org Syn 1988;VI:301-2.

47. Cason J. $\beta$-Methylglutaric anhydride. Org Syn 1963;IV:630-1.

48. Farmer HH, Rebjohn N. $\beta$-Ethyl- $\beta$-methylglutaric acid. Org Syn 1963;IV:441-2.

49. Cason J. $\beta$-Carbomethoxypropionyl chloride. Org Syn 1955;III: 169-70.

50. Vogel AI. Vogel's text book of practical organic chemistry. 5th ed. Harlow: Longman Group, UK Limited; 1989.

51. Strube RE. Ethyl tert-butyl malonate. Org Syn 1963;IV:417-18.

52. Paquet LA, ed. Encyclopedia of reagents for organic synthesis. Vol. 7. Pennsylvania State University, John-Wiley; 1995. Available from: http://eu.wiley.com/WileyCDA/WileyTitle/productCd-0470017546. html [last accessed 21 Jul 2015].

53. Krapcho AP, Diamanti J, Cayen C, Bingham R. 2Carbethoxycyclooctanone. Org Syn 1973;V:198-9.

54. Popović-Djordjević JB, Došen-Mićović LjI, Juranić IO, Drakulić BJ. Antiproliferative activity of NCI-DTP glutarimide derivatives: an alignment independent 3D QSAR study. J Serbian Chem Soc 2010; 75:1167-79.

55. Cruciani G, ed. Methods and principles in medicinal chemistry, molecular interaction fields: applications in drug discovery and ADME prediction. Weinheim: Wiley-VCH; 2006. 